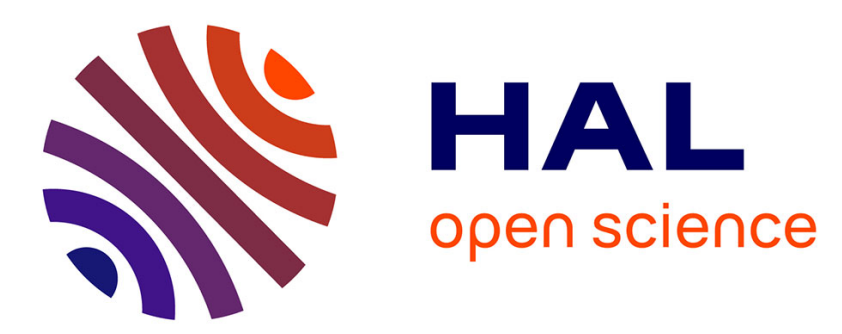

\title{
Geometric Determination of the Cable-Cylinder Interference Regions in the Workspace of a Cable-Driven Parallel Robot
}

\author{
Antoine Martin, Stéphane Caro, Philippe Cardou
}

\section{To cite this version:}

Antoine Martin, Stéphane Caro, Philippe Cardou. Geometric Determination of the Cable-Cylinder Interference Regions in the Workspace of a Cable-Driven Parallel Robot. The Third International Conference on Cable-Driven Parallel Robots (CableCon 2017), Aug 2017, Québec, Canada. hal01757799

\author{
HAL Id: hal-01757799 \\ https://hal.science/hal-01757799
}

Submitted on 4 Apr 2018

HAL is a multi-disciplinary open access archive for the deposit and dissemination of scientific research documents, whether they are published or not. The documents may come from teaching and research institutions in France or abroad, or from public or private research centers.
L'archive ouverte pluridisciplinaire HAL, est destinée au dépôt et à la diffusion de documents scientifiques de niveau recherche, publiés ou non, émanant des établissements d'enseignement et de recherche français ou étrangers, des laboratoires publics ou privés. 


\title{
Geometric Determination of the Cable-Cylinder Interference Regions in the Workspace of a Cable-Driven Parallel Robot
}

\author{
Antoine Martin, Stéphane Caro, Philippe Cardou
}

\begin{abstract}
Cable-Driven Parallel Robots (CDPRs) are a type of parallel robots that have the particularity of using cables as legs. CDPRs have several advantages such as large workspaces, high acceleration and high payload capacity. However, CDPRs present also some drawbacks such as the possible collisions between their cables and environment. Therefore, this paper is about the geometric determination of the cable-cylinder interference regions in the workspace of a CDPR. The cables are considered massless and straight. Then, the boundaries of the interference regions onto the cylinder form a closed loop composed of arcs and straight line segments that can be expressed symbolically. Those geometric entities generate truncated cones and planes corresponding to the boundaries of the volume of interferences. Finally, a methodology is described to trace the cable-cylinder interference free constant orientation workspace of CDPRs.
\end{abstract}

\section{Introduction}

Cable-driven parallel robots (CDPRs) have received increasing attention from researchers during the last 20 years, in part because of their large workspace and low mass in motion. CDPR workspaces such as the Wrench Closure Workspace $[1,2]$ or the Wrench Feasible Workspace [3] have been widely studied. These workspaces

\footnotetext{
Antoine Martin

École Centrale de Nantes-Laboratoire des Sciences du Numérique de Nantes, 1, rue de la Noë, BP 92101, 44321 NANTES Cedex 3, e-mail: antoine.martin@1s2n.fr

Stéphane Caro

CNRS-Laboratoire des Sciences du Numérique de Nantes, 1, rue de la Noë, BP 92101, 44321

NANTES Cedex 3, e-mail: stephane.caro@1s2n.fr, stephane.caro@irccyn.ec-nantes.fr

Philippe Cardou

Université Laval, 1065 Avenue de la médecine Québec, Québec G1V 0A6 Canada e-mail: pcardou@gmc.ulaval.ca
} 
allow the visualisation of the volume over which the moving-platform can sustain certain external wrenches. The Twist Feasible Workspace [4] and the Dynamic Feasible Workspace [5] have also been defined in the literature in order to evaluate the capability of a CDPR to perform required twists and accelerations.

One of the main drawbacks of CDPRs is the potential risk of collisions (also called interferences) between their cables and their environment. Although such collisions may occur in many industrial operations, there have been few papers in the literature dealing with this issue. Moreover interferences usually reduce the size of the CDPR workspace [6]. For instance, for industrial operations over a large workspace and in a cluttered environment such as that shown in Fig. 1, the workspace of the CDPR is dramatically reduced because of the possible collisions between the cables and the tubes of the large truss [7]. Another relevant application is detailed in reference [8], where two CDPRs are working side by side, and collisions between cables can occur. In [9], four types of interferences are defined:

1. Collisions between two cables.

2. Collisions between a cable and the platform.

3. Collisions between a cable and the environment.

4. Collisions between the platform and the environment.

The first two types of collisions were studied in $[9,10]$ for a moving-platform described by a set of triangles. A geometric method to compute the interference-free constant-orientation workspace of CDPRs was introduced in [11] while considering the cable-cable and cable-platform interferences. This method has been used to develop the ARACHNIS interface [12], which aims to help robot designers trace CDPR workspaces and find optimal dimensions for their good under design. In the foregoing works, cables have been considered massless and straight. A recent paper from Wang et al. [13] describes an algorithm to trace the Collisions Free Force

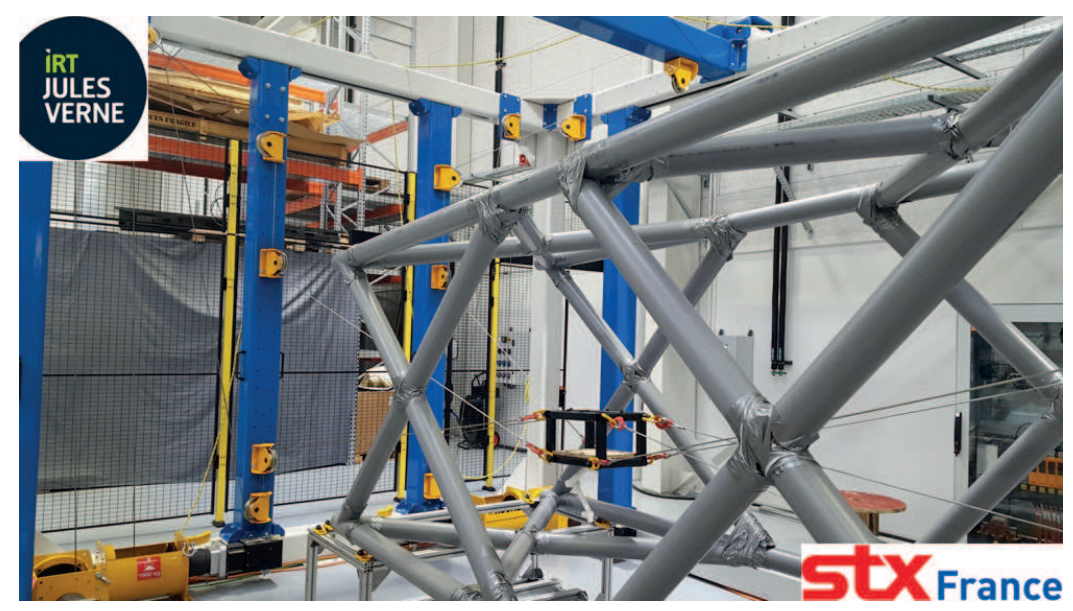

Fig. 1 CAROCA prototype: a reconfigurable cable-driven parallel robot working in a cluttered environment (Courtesy of IRT Jules Verne and STX France) 
Closure Workspace of a CDPR working in a cluttered environment. This algorithm, which uses the convex hull approach, turns out to be time consuming, mainly when the cluttered environment is known beforehand.

This paper deals with the geometric determination of cable-cylinder interference regions in the CDPR workspace. A mathematical description of the boundaries of those regions is obtained for a constant orientation of the moving-platform. The paper is organized as follows. Section 2 deals with the determination of interference region between one cable and a cylinder. Section 3 describes the method proposed to find the interference-free constant-orientation workspace of a cable-driven parallel robot while considering the collisions between its cables and a cylinder. Finally, the determination of the interference regions between the cables of the CAROCA prototype [7] and a cylinder highlights the contributions of this paper.

\section{Interference region between a cable and a cylinder}

Due to their large workspaces, CDPRs can be used to perform maintenance operations on large structures such as bridges. For instance, Fig. 1 shows a CPDR working inside a tubular truss. In those cases, interferences between the CDPR cables and the cylinders forming the structure are the main limitation on the workspace.

\subsection{Parametrisation}

As shown in Fig. 2, the $i$ th cable of a CDPR is attached to the moving-platform at anchor point $B_{i}$. Its length is controlled by an actuated reel whose exit point $A_{i}$ is fixed to the base. The base frame is denoted $\mathscr{F}_{b}=\left(O, \mathbf{x}_{b}, \mathbf{y}_{b}, \mathbf{z}_{b}\right)$. Frame $\mathscr{F}_{p}=$ $\left(P, \mathbf{x}_{p}, \mathbf{y}_{p}, \mathbf{z}_{p}\right)$ is attached to the moving-platform. The cylinder $\mathscr{C}$ with base point $C$, radius $r_{c}$, length $l_{c}$ and axis $\mathscr{A}_{C}$ is located inside the working area of a CDPR, fixed to its base.

This section aims at determining for a given orientation of the moving-platform, the positions of $P$ that lead to collisions between cable $\mathscr{C}_{i}$ and cylinder $\mathscr{C}$. Here, the cable is assumed to be straight, with a negligible cross-section. The interference point between cable $\mathscr{C}_{i}$ and cylinder $\mathscr{C}$ is named $I$. $\mathbf{p}_{c, i}$ denotes the Cartesian coordinate vector of point $I$ expressed in the base frame $\mathscr{F}_{b}$.

The proposed strategy for determining and tracing the region of interference between $\mathscr{C}_{i}$ and $\mathscr{C}$ consists in moving point $P$ while maintaining contact $I$ between the line segment and the cylinder. In doing so, point $P$ sweeps a conical surface in space, while point $I$ makes a closed loop on the surface of $\mathscr{C}$. Interferences between $\mathscr{C}_{i}$ and $\mathscr{C}$ can occur in two ways: either the interference point $I$ lies on the cylindrical surface of $\mathscr{C}$, either it lies on one of its two circular edges. 


\subsection{Boundaries of the cable-cylinder interference region}

The type of closed-loop trajectory followed by $I$ through this sweeping motion depends on the location of $A_{i}$ with respect to $\mathscr{C}$. One finds five zones for the location of $A_{i}$, which correspond to three types of closed loop trajectories of $I$ over the surface of $\mathscr{C}$. Figure 3 represents a section of the top half of the cylinder with the five zones in question. Its bottom half is the mirror image of the top one with respect to axis $\mathscr{A}_{C}$.

It is noteworthy that the types of point $I$ trajectories when exit point $A_{i}$ belongs to zones $\mathscr{Z}_{2}^{\prime}$ and $\mathscr{Z}_{3}^{\prime}$ are the same as when exit point $A_{i}$ belongs to zones $\mathscr{Z}_{2}$ and $\mathscr{Z}_{3}$, respectively. For zones $\mathscr{Z}_{1}$ and $\mathscr{Z}_{2}$, the interference regions between the $i$ th cable $\mathscr{C}_{i}$ and the cylindrical part of $\mathscr{C}$ are considered. Then, the interference regions between $\mathscr{C}_{i}$ and the endcaps of $\mathscr{C}$ are obtained. For zone $\mathscr{Z}_{3}$, only this second part is needed.

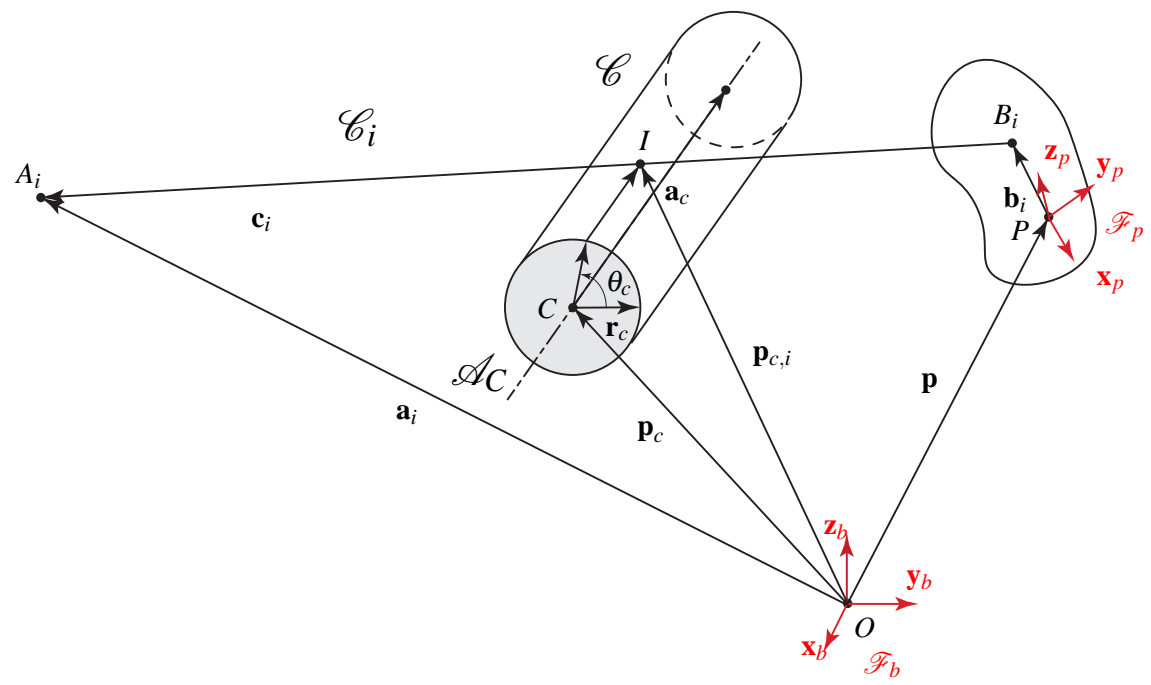

Fig. 2 th cable $\mathscr{C}_{i}$, CDPR moving-platform and cylinder $\mathscr{C}$

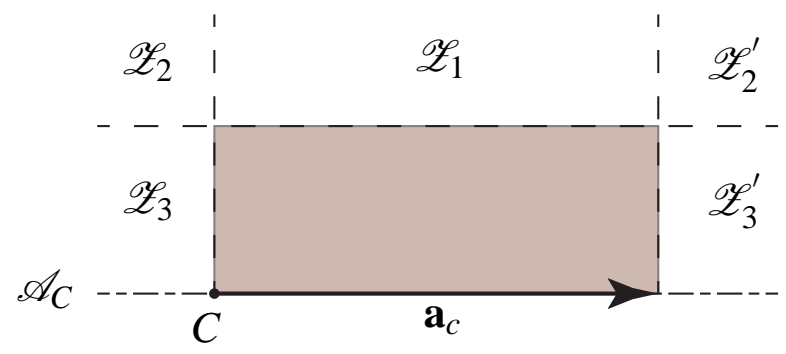

Fig. 3 Position of exit point $A_{i}$ relatively to cylinder $\mathscr{C}$ 


\subsubsection{Interferences between cable $\mathscr{C}_{i}$ and the cylindrical part of $\mathscr{C}$}

The boundaries of the interference region between cable $\mathscr{C}_{i}$ and the cylindrical part of cylinder $\mathscr{C}$ describe the points where a straight line coming from exit point $A_{i}$ is tangent to the cylinder $\mathscr{C}$. As the interference point $I$ belongs to the cylinder $\mathscr{C}$, we can write:

$$
\mathbf{p}_{c, i}=\mathbf{p}_{c}+\alpha_{c} \mathbf{a}_{c}+\mathbf{Q}\left(\mathbf{a}_{c}, \theta_{c}\right) \mathbf{r}_{c}
$$

where $\mathbf{p}_{c}$ is the Cartesian coordinate vector of point $C$, depicted in Fig. 2, $\alpha_{c}$ is a scalar bounded between 0 and 1 and represents the location of point $I$ along the cylinder axis $\mathscr{A}_{C} \cdot \mathbf{r}_{C}$ is a vector normal to axis $\mathscr{A}_{C}$. Therefore, the following equation holds:

$$
\mathbf{a}_{c}^{T} \mathbf{r}_{c}=0
$$

$\mathbf{Q}\left(\mathbf{a}_{c}, \theta_{c}\right)$ is the rotation matrix of vector $\mathbf{r}_{c}$ about $\mathscr{A}_{C}$ by an angle $\theta_{c}$.

Accordingly, the tangency condition between the $i$ th cable $\mathscr{C}_{i}$ and the cylindrical part of cylinder $\mathscr{C}$ is expressed as:

$$
\left(\mathbf{p}_{c, i}-\mathbf{a}_{i}\right)^{T}\left(\mathbf{Q}\left(\mathbf{a}_{c}, \theta_{c}\right) \mathbf{r}_{c}\right)=0
$$

From Eqs. (2) and (3), angle $\theta_{c}$ can take two values, namely,

$$
\left\{\begin{array}{l}
\mathbf{Q}\left(\mathbf{a}_{c}, \theta_{c}^{+}\right) \mathbf{r}_{c}=\eta_{c} \mathbf{a}_{c} \times\left(\mathbf{p}_{c, i}-\mathbf{a}_{i}\right), \\
\mathbf{Q}\left(\mathbf{a}_{c}, \theta_{c}^{-}\right) \mathbf{r}_{c}=-\eta_{c} \mathbf{a}_{c} \times\left(\mathbf{p}_{c, i}-\mathbf{a}_{i}\right) .
\end{array}\right.
$$

where $\eta_{c}=\frac{r_{c}}{\left\|\mathbf{a}_{c} \times\left(\mathbf{p}_{c, i}-\mathbf{a}_{i}\right)\right\|_{2}}$. For the sake of conciseness, $\mathbf{Q}\left(\mathbf{a}_{c}, \theta_{c}^{+}\right) \mathbf{r}_{c}$ and $\mathbf{Q}\left(\mathbf{a}_{c}, \theta_{c}^{-}\right) \mathbf{r}_{c}$ are denoted as $\mathbf{r}_{c}^{+}$and $\mathbf{r}_{c}^{-}$, respectively. Therefore $\theta_{c}^{+}$and $\theta_{c}^{-}$take the form:

$$
\left\{\begin{array}{l}
\theta_{c}^{+}=\arccos \left(\frac{\mathbf{r}_{c}^{T} \mathbf{r}_{c}^{+}}{\left\|\mathbf{r}_{c}\right\| \cdot\left\|\mathbf{r}_{c}^{+}\right\|}\right) \\
\theta_{c}^{-}=\arccos \left(\frac{\mathbf{r}_{c}^{T} \mathbf{r}_{c}^{-}}{\left\|\mathbf{r}_{c}\right\| \cdot\left\|\mathbf{r}_{c}^{-}\right\|}\right)
\end{array}\right.
$$

Upon introducing the position vector of point $I$ of Eq. (1) into Eq. (4), we obtain:

$$
\left\{\begin{array}{l}
\mathbf{r}_{c}^{+}=\eta_{c} \mathbf{A}_{c}\left(\mathbf{p}_{c, i}+\alpha_{c} \mathbf{a}_{c}+\mathbf{r}_{c}^{+}-\mathbf{a}_{i}\right), \\
\mathbf{r}_{c}^{-}=-\eta_{c} \mathbf{A}_{c}\left(\mathbf{p}_{c, i}+\alpha_{c} \mathbf{a}_{c}+\mathbf{r}_{c}^{-}-\mathbf{a}_{i}\right) .
\end{array}\right.
$$

$\mathbf{A}_{c}$ being the cross-product matrix ${ }^{1}$ of vector $\mathbf{a}_{c}$. By solving Eq. (6), $\mathbf{r}_{c}^{+}$and $\mathbf{r}_{c}^{-}$take the form:

$$
\left\{\begin{array}{l}
\mathbf{r}_{c}^{+}=\frac{\eta_{c}}{1+l_{c}^{2}}\left(r_{c} \mathbf{A}_{c}^{2}+\mathbf{A}_{c}\right)\left(\mathbf{p}_{c, i}-\mathbf{a}_{i}\right), \\
\mathbf{r}_{c}^{-}=\frac{\eta_{c}}{1+l_{c}^{2}}\left(r_{c} \mathbf{A}_{c}^{2}-\mathbf{A}_{c}\right)\left(\mathbf{p}_{c, i}-\mathbf{a}_{i}\right) .
\end{array}\right.
$$

\footnotetext{
${ }^{1}$ The cross-product matrix $\mathbf{Y}$ of $\mathbf{y}$ is defined as $\delta(\mathbf{y} \times \mathbf{x}) \backslash \delta \mathbf{x}$ for any $\mathbf{x}, \mathbf{y} \in \mathbb{R}^{3}$.
} 
As a result, the boundaries of the interference region between cable $\mathscr{C}_{i}$ and the cylindrical part of $\mathscr{C}$ can be expressed as:

$$
\left\{\begin{array}{l}
\mathbf{p}_{c, i}^{+}=\mathbf{p}_{c}+\alpha_{c} \mathbf{a}_{c}+\mathbf{r}_{c}^{+} \\
\mathbf{p}_{c, i}^{-}=\mathbf{p}_{c}+\alpha_{c} \mathbf{a}_{c}+\mathbf{r}_{c}^{-}
\end{array}\right.
$$

with $\alpha_{c}$ being a scalar lying between 0 and 1 . The edges of the corresponding line segments are named $I_{n}^{+}$and $I_{n}^{-}$for $\alpha_{c}=0$, and $I_{f}^{+}$and $I_{f}^{-}$for $\alpha_{c}=1$ as illustrated in Fig. 4.

\subsubsection{Interferences with the endcaps}

For the second point, the interferences with the endcaps of the cylinder are studied. The question is to find how to connect $I_{n}^{+}$to $I_{n}^{-}$and $I_{f}^{+}$to $I_{f}^{-}$. Those connections change depending on the zone to which $A_{i}$ belongs to.

In $\mathscr{Z}_{1}$, the curve linking $I_{n}^{+}$to $I_{n}^{-}$is an arc starting from $I_{n}^{-}$and going to $I_{n}^{+}$. The result is the same for $I_{f}^{+}$and $I_{f}^{-}$. We obtain the equations:

$$
\left\{\begin{array}{l}
\mathbf{p}_{c, i}^{n}=\mathbf{p}_{c}+\mathbf{Q}\left(\mathbf{a}_{c}, \theta_{c}\right) \mathbf{r}_{c}^{-}, \\
\mathbf{p}_{c, i}^{f}=\mathbf{p}_{c}+\mathbf{a}_{c}+\mathbf{Q}\left(\mathbf{a}_{c}, \theta_{c}\right) \mathbf{r}_{c}^{-} .
\end{array}\right.
$$

with $\theta_{c}$ lying in the interval $\left[0, \theta_{c}^{+}-\theta_{c}^{-}\right]$. The result is shown in Fig. $5(a)$.

For $\mathscr{Z}_{2}$, the behavior is different for the nearest endcap. This time, the arc to take into account starts from $I_{n}^{+}$and goes to $I_{n}^{-}$. The equations become:

$$
\begin{cases}\mathbf{p}_{c, i}^{n}=\mathbf{p}_{c}+\mathbf{Q}\left(\mathbf{a}_{c}, \theta_{c}\right) \mathbf{r}_{c}^{+} & \theta_{c} \in\left[0,2 \pi-\left(\theta_{c}^{+}-\theta_{c}^{-}\right)\right] \\ \mathbf{p}_{c, i}^{f}=\mathbf{p}_{c}+\mathbf{a}_{c}+\mathbf{Q}\left(\mathbf{a}_{c}, \theta_{c}\right) \mathbf{r}_{c}^{-} & \theta_{c} \in\left[0, \theta_{c}^{+}-\theta_{c}^{-}\right]\end{cases}
$$

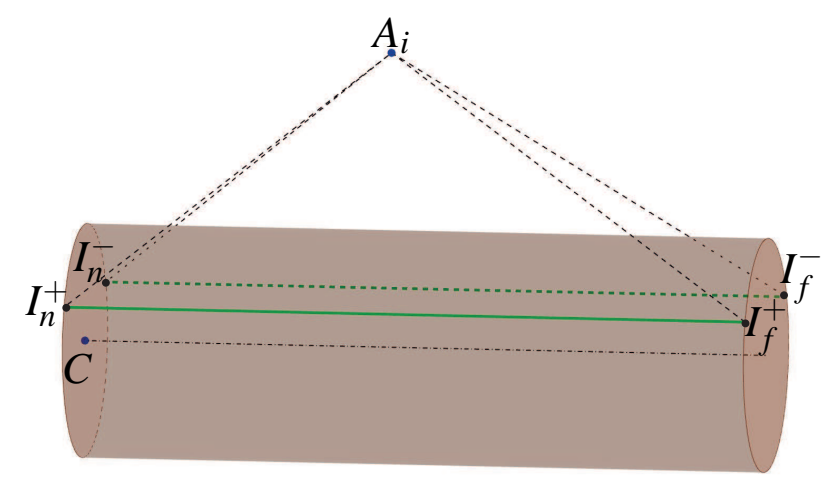

Fig. 4 Boundaries of the interference region between cable $\mathscr{C}_{i}$ and the cylindrical part of $\mathscr{C}$ 


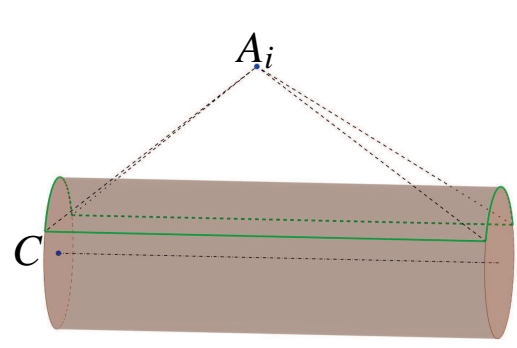

(a)

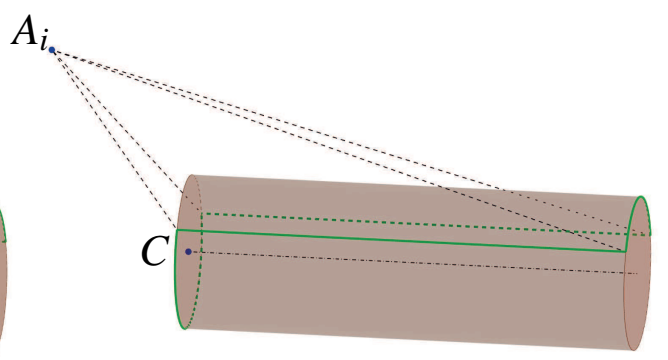

(b)

Fig. 5 Boundaries in (a) $\mathscr{Z}_{1}$ and $(b) \mathscr{Z}_{2}$

The result is shown in Fig. $5(b)$.

When $A_{i}$ is in $\mathscr{Z}_{3}$, the tangency condition of Eq. (3) is no longer satisfied. In that case, the boundaries of the interference volume for the entire cylinder is the same as the one with the nearest endcap alone. The result is the circle defined by:

$$
\mathbf{p}_{c, i}^{n}=\mathbf{p}_{c}+\mathbf{Q}\left(\mathbf{a}_{c}, \theta_{c}\right) \mathbf{r}_{c}, \quad \theta_{c} \in[0,2 \pi]
$$

In each case, the boundaries are either straight lines or arcs. The interference volume obtained from those geometric forms are detailed in the following section.

\subsection{Interferences with straight line segments and arcs}

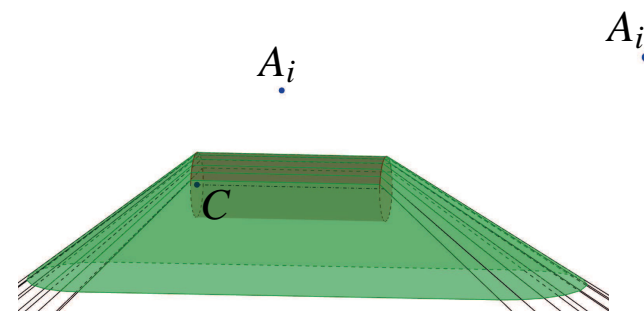

(a)

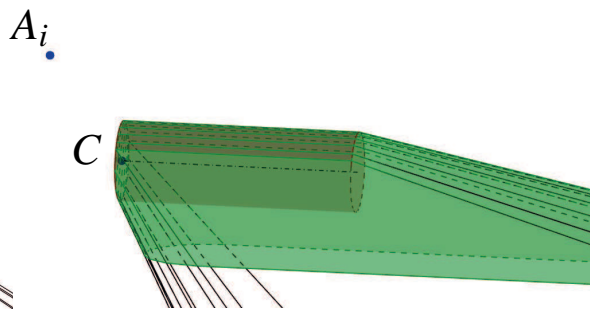

(b)

Fig. 6 Interference regions when $A_{i}$ is in $(a) \mathscr{Z}_{1}$ and $(b) \mathscr{Z}_{2}$

As seen above, from point $A_{i}$, the closed-loop trajectory followed by $I$ is composed of straight line and arc segments. Since a cable is considered straight without deformation, a line coming from $A_{i}$ and following this shape will draw the external boundary of the interference volume. 
In the case of the straight line between $I_{n}^{+}$and $I_{f}^{+}\left(I_{n}^{-}\right.$and $I_{f}^{-}$respectively), the boundary is the infinite triangle originating from $A_{i}$ and passing by those two points. Since the extremity $B_{i}$ of the cable needs to be after the cylinder for having a possible collision, this triangle is truncated by the segment $\left[I_{n}^{+} I_{f}^{+}\right]$.

For the arc between $I_{n}^{+}$and $I_{n}^{-}\left(I_{f}^{+}\right.$and $I_{f}^{-}$respectively), the result will be an oblique cone, truncated twice:

- Once by the plane defined by points $A_{i}, I_{n}^{+}$and $I_{n}^{-}$.

- Once by the plane defined by points $C, I_{n}^{+}$and $I_{n}^{-}$.

Finally, the part of the cylinder closest to $A_{i}$ and inside the lines detailed above need to be taken into account, to close the surface delimiting the interference volume. This part of the cylinder is easily obtained from the results of Sec. 2.2. Two examples are shown in Fig. 6 when the exit point of the winch is in zone $\mathscr{Z}_{1}$, and in zone $\mathscr{Z}_{2}$.

\section{Interferences between the cables of a CDPR and a cylinder}

In this section, the geometric model of a CDPR is detailed in Sec. 3.1. The interference volume between one cable and a cylinder obtained in Sec. 2 can then be coupled with this model to obtain in Sec. 3.2 the positions of the platform for which at least one cable is in collision with the cylinder.

\subsection{Geometric modeling}

In Fig. 2, one can see the platform of a CDPR with cable $\mathscr{C}_{i}$ attached to it at point $B_{i}$. The exit point of the winch controlling its length is $A_{i}$. From this, by considering the cable as a straight line, a loop-closure equation gives the following geometric model:

$$
\mathbf{c}_{i}=\mathbf{a}_{i}-\mathbf{p}-\mathbf{R} \mathbf{b}_{i}
$$

where $\mathbf{R}$ refers to the rotation matrix that transforms the global frame $\mathscr{F}_{b}$ to the frame attached to the mobile platform $\mathscr{F}_{p}$.

\subsection{Interferences}

Since the interference point between cable $\mathscr{C}_{i}$ and the cylinder belongs to both objects, its position can be expressed either by Eq. (1) or by:

$$
\mathbf{p}_{c, i}=\mathbf{p}+\mathbf{R} \mathbf{b}_{i}+\gamma_{i} \mathbf{c}_{i}
$$


where $\gamma_{i}$ is an scalar bounded between 0 and 1 defining the position of the interference point. 0 means that the collision occurs at point $B_{i}, 1$ in means that it happens at point $A_{i}$. The combination of Eqs. (1) and (13) leads the following equation:

$$
\mathbf{p}+\mathbf{R} \mathbf{b}_{i}+\gamma_{i} \mathbf{c}_{i}=\mathbf{p}_{c}+\alpha_{c} \mathbf{a}_{c}+\mathbf{Q}\left(\mathbf{a}_{c}, \theta_{c}\right) \mathbf{r}_{c}
$$

Upon substituting the expression of $\mathbf{c}_{i}$ defined in Eq. (12) into Eq. (14), we obtain:

$$
\mathbf{p}=\mathbf{a}_{i}-\mathbf{R} \mathbf{b}_{i}+\frac{1}{1-\gamma_{i}}\left(\mathbf{p}_{c}-\mathbf{a}_{i}\right)+\frac{1}{1-\gamma_{i}}\left(\alpha_{c} \mathbf{a}_{c}+\mathbf{Q}\left(\mathbf{a}_{c}, \theta_{c}\right) \mathbf{r}_{c}\right) .
$$

This equation is valid for $\gamma_{i} \neq 1$. It is the case when the collision happens at the exit point of the winch, which can easily be avoided at the design phase since this point is not moving. By changing the values of $\alpha_{c}$ and $\theta_{c}$ according to Sec. 2, we obtain the volume in which the position $\mathbf{p}$ of the platform generates an interference with cable $\mathscr{C}_{i}$. This method needs to be applied for each cable-cylinder combination to obtain the interference region of the entire CDPR.

\subsection{CAROCA prototype-cylinder interference region}

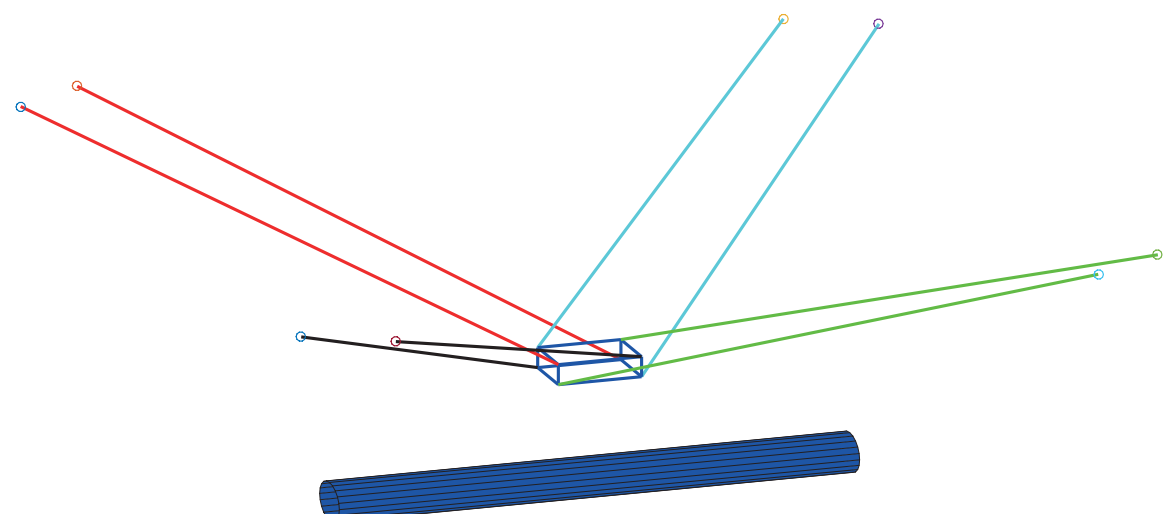

Fig. 7 One configuration of the reconfigurable CAROCA prototype and a cylinder in its working area

Gagliardini et al. [7] introduced a method to manage the discrete reconfigurations of a CDPR, in order to use the latter for sandblasting and painting the outer part and the inner part of a large tubular structure with a minimal number of reconfigurations. This is a typical cluttered environment for which the method presented in this paper should help simplify the reconfiguration planning of the CDPR.

Figure 7 represents a CDPR with one of the configurations obtained in [7]. The 


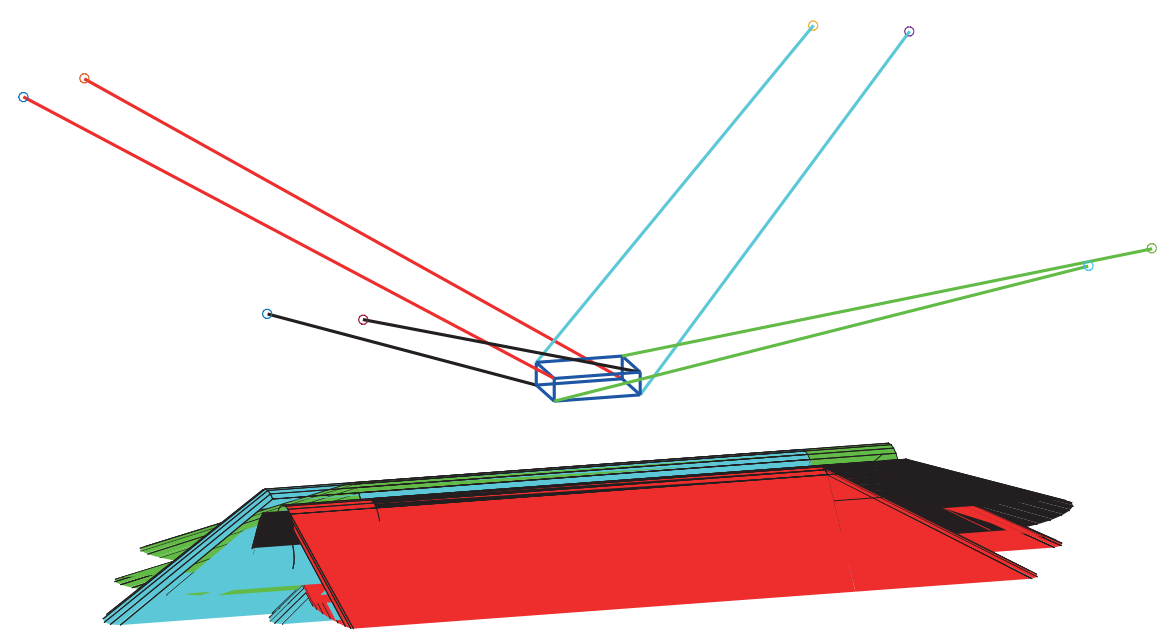

Fig. 8 Locations of the geometric center of the CAROCA mobile platform leading to some cablecylinder interferences

tubular structure is replaced with a single cylinder, to simplify the analysis of the interference regions. The results are shown in Fig. 8. For the cylinder included into the working area of the CDPR, eight interferences regions, namely one for each cable, are obtained. It is noteworthy that those regions are valid for a constant orientation of the mobile-platform.

\section{Conclusion}

The paper dealt with the geometric determination of the cable-cylinder interference regions in the workspace of a CDPR. First, a methodology was described to determine in a closed-form the boundaries of the interference region between a cable and a cylinder. The latter is first split into three parts, its two endcaps and the rest of the cylinder. By considering the cable tangent to the cylinder on the boundaries of the interference region, four points are obtained. Those points are connected by a straight segment along the cylinder, and two arcs along its endcaps depending on the position of the cable exit point with respect to the cylinder. Five zones for this position are defined, each one corresponds to a different arc segment to be taken into account to draw the boundaries of the interference region. Those line and arc segments generate truncated planes and oblique cones shapes, forming the boundaries of the interference volume with a part of the cylinder. Then, the cable-cylinder interference free constant orientation workspace of a cable-driven parallel robot can be traced while considering one or several cylinder(s) within the working area. As an illustrative example, the cable-cylinder interference free constant orientation workspace of the reconfigurable CAROCA prototype has been traced while considering a cylinder in 
its environment. An add-on feature to trace the cable-cylinder interference free constant orientation workspace of any cable-driven parallel robot will be implemented in ARACHNIS software [12] in future work. Finally, the contributions of this paper should ease the design of new cable-driven parallel robots working in a cluttered environment.

Acknowledgements The financial support of the RFI ATLANSTIC 2020 CREATOR project is greatly acknowledged.

\section{References}

1. Marc Gouttefarde and Clément M. Gosselin. On the properties and the determination of the wrench-closure workspace of planar parallel cable-driven mechanisms. In ASME 2004 International Design Engineering Technical Conferences and Computers and Information in Engineering Conference, pages 337-346. American Society of Mechanical Engineers, 2004.

2. Ethan Stump and Vijay Kumar. Workspaces of Cable-Actuated Parallel Manipulators. Journal of Mechanical Design, 128(1):159, 2006.

3. Marc Gouttefarde, Jean-Pierre Merlet, and David Daney. Wrench-feasible workspace of parallel cable-driven mechanisms. In Robotics and Automation, 2007 IEEE International Conference on, pages 1492-1497. IEEE, 2007.

4. Lorenzo Gagliardini, Stéphane Caro, and Marc Gouttefarde. Dimensioning of cable-driven parallel robot actuators, gearboxes and winches according to the twist feasible workspace. In 2015 IEEE International Conference on Automation Science and Engineering (CASE), pages 99-105. IEEE, 2015.

5. Guillaume Barrette and Clément M. Gosselin. Determination of the Dynamic Workspace of Cable-Driven Planar Parallel Mechanisms. Journal of Mechanical Design, 127(2):242, 2005.

6. Robert L. Williams and Paolo Gallina. Planar cable-direct-driven robots, part i: Kinematics and statics. In Proceedings of the 2001 ASME Design Technical Conference, 27th Design Automation Conference, pages 178-186, 2001.

7. Lorenzo Gagliardini, Stéphane Caro, Marc Gouttefarde, and Alexis Girin. Discrete reconfiguration planning for Cable-Driven Parallel Robots. Mechanism and Machine Theory, 100:313337, June 2016.

8. Martin J.-D. Otis, Simon Perreault, Thien-Ly Nguyen-Dang, Patric Lambert, Marc Gouttefarde, Denis Laurendeau, and Clément Gosselin. Determination and Management of Cable Interferences Between Two 6-DOF Foot Platforms in a Cable-Driven Locomotion Interface. IEEE Transactions on Systems, Man, and Cybernetics - Part A: Systems and Humans, 39(3):528-544, May 2009.

9. Dinh Quan Nguyen and Marc Gouttefarde. On the Improvement of Cable Collision Detection Algorithms. In Andreas Pott and Tobias Bruckmann, editors, Cable-Driven Parallel Robots, number 32 in Mechanisms and Machine Science, pages 29-40. Springer International Publishing, 2015. DOI: 10.1007/978-3-319-09489-2_3.

10. Jean-Pierre Merlet. Analysis of the influence of wires interference on the workspace of wire robots. In On Advances in Robot Kinematics, pages 211-218. Springer, 2004.

11. Simon Perreault, Philippe Cardou, Clément M. Gosselin, and Martin J.-D. Otis. Geometric Determination of the Interference-Free Constant-Orientation Workspace of Parallel CableDriven Mechanisms. Journal of Mechanisms and Robotics, 2(3):031016, 2010.

12. Ana Lucia Cruz Ruiz, Stéphane Caro, Philippe Cardou, and Franois Guay. Arachnis: Analysis of robots actuated by cables with handy and neat interface software. In Cable-Driven Parallel Robots, pages 293-305. Springer, 2015. 
13. Bingyao Wang, Bin Zi, Sen Qian, and Dan Zhang. Collision free force closure workspace determination of reconfigurable planar cable driven parallel robot. In 2016 Asia-Pacific Conference on Intelligent Robot Systems (ACIRS), pages 26-30, July 2016.

14. Laurent Blanchet. Contribution à la modélisation de robots à câbles pour leur commande et leur conception. PhD thesis, Université Nice Sophia Antipolis, 2015. 